\title{
A family of groups with a countable infinity of full orders
}

\author{
R. N. Buttsworth
}

We construct a family of groups with precisely $\aleph_{0}$ full orders.

\section{Introduction}

In Fuchs [1], B.H. Neumann is reported as asking if, when a orderable group has infinitely many full orders, the total number of these is a power of 2 . We show that there are groups with precisely a countable infinity of orders.

\section{Notation and preliminary results}

Group operations are written multiplicatively. Elementary results about ordered groups are assumed and both these and relevant notation is found in Fuchs [1]. The following easy results are also assumed.

Let $G$ be a fully ordered group with order denoted by $\succ$ and

$$
x, y_{i} \in G, \quad i \in z \text {. }
$$

2.1. If

$$
x^{-1} y_{i}^{k_{i}} x=y_{i}^{z_{i}}, \quad k_{i}, z_{i} \in z, k_{i} \neq z_{i},
$$

then

$$
|x| \gg\left|y_{i}\right|
$$

and

Receíved 8 September 1970. 


$$
\left|y_{i}\right| \sim\left|y_{j}\right| \text { if } k_{i} z_{j}=k_{j} z_{i} \text {. }
$$

2.2. If $x^{-1} y_{i} x=y_{i+1}$ and if $\left|y_{i}\right| \sim\left|y_{i+1}\right|$ does not hold then either

$$
\left.\left.|x| \gg \ldots \gg\left|y_{i+1}\right| \gg\right\rangle\left|y_{i}\right| \gg\left|y_{i-1}\right| \gg\right\rangle \ldots
$$

or

$$
\left.\left.\left.|x| \gg\rangle \ldots \succ\left|y_{i-1}\right| \gg\right\rangle\left|y_{i}\right| \succ\right\rangle\left|y_{i+1}\right| \succ\right\rangle \ldots .
$$

\section{The family of groups}

Our main result is:

THEOREM 3.1. There are 0-groups with precisely a countable infinity of distinct fuzl orders.

We prove this by producing a family of such groups. First we need a few definitions.

DEFINITION 3.2. $X$ is that subgroup of the rational numbers under addition whose elements are just those with denominators a power of 2 .

DEFINITION 3.3. $X_{1}$ is a subset of $X$ given by

$$
X_{1}=\{x \mid x \in X, 0 \leq x<1\}
$$

so that

$$
X_{1}=\left\{\frac{m}{2^{n}} \mid m<2^{n}, m, n \in N\right\} \cup\{0\},
$$

where $N$ denotes the positive integers.

$Z$ is the integers under addition.

DEFINITION 3.4. The groups $H_{z, x}$ and $K_{z, x}: z \in Z, x \in X_{1}$ are all copies of $Q$, the rational numbers under addition.

DEFINITION 3.5 .

$$
H_{z}=\prod_{x \in X_{1}} H_{z, x}
$$

and 


$$
K_{z}=\prod_{x \in X_{1}} K_{z, x},
$$

are (restricted) direct products of copies of $Q$.

$$
\begin{aligned}
& H=\prod_{z \in Z} H_{z}=\prod_{z \in Z} \prod_{x \in X_{1}} H_{z, x}, \\
& K=\prod_{z \in Z} K_{z}=\prod_{z \in Z} \prod_{x \in X_{1}} K_{z, x},
\end{aligned}
$$

and

$$
L=H \times K \text {. }
$$

Next we define a semidirect product of $X$ and $L$. To accomplish this we specify the transformations of the basic components, $H_{z, x}$ and $K_{z, x}$ by each element of $X$.

DEFINITION 3.6. Let $h_{z, x} \in, H_{z, x}$ be a distinguished element for each $H_{z, x}$ and similarly

$$
k_{z, x} \in K_{z, x}
$$

Likewise $\xi \in X$ is distinguished and so is $\zeta \in Z$.

Thus arbitrary members of $H_{z, x}$ and of $X$ can be expressed as $h_{z, x}^{r}$ and $\xi^{\alpha}$ respectively, where $r \in Q$ and $\alpha \in X$.

Our transformations are given by

$$
\xi^{-\alpha} h_{z, x^{x}} \xi^{\alpha}=h_{z, x+\alpha 2^{2}-n}
$$

where $n \leq x+\alpha 2^{z}<n+1, n \in N$, and

$$
\xi^{-\alpha} k_{z, x^{r}} \xi^{\alpha}=k^{r q^{n}}{ }_{z, x+\alpha 2^{2}-n}
$$

where

$$
n \leq x+\alpha 2^{z}<n+1
$$


and where $p, q \in N$ are square-free with

$$
p \neq q \text {. }
$$

LEMMA 3.7. These transformations form a subgroup of the automorphism group of $L$ isomorphic to $X$, so we have an associated semidirect product, $M$, of $L$ by $X$.

Finally we define transformations of $M$ by $Z$.

DEFINITION 3.8 .

$$
\begin{aligned}
& \zeta^{-\beta} h_{z, x^{r}} \zeta^{\beta}=h_{z+\beta, x}^{r}, \\
& \zeta^{-\beta} k_{z, x^{r}} \zeta^{\beta}=k_{z+\beta, x}^{r},
\end{aligned}
$$

and

$$
\zeta^{-\beta} \xi^{\alpha} \zeta^{\beta}=\xi^{\frac{\alpha}{2^{\beta}}} .
$$

LEMMA 3.9. These transformations form a subgroup of the automorphism group of $M$ isomorphic to $Z$, so we have an associated semidirect product, $G(p, q)$ of $M$ by $Z$.

LEMMA 3.10. In any fuzz order of $M$ (and hence of $G(p, q)$ ) the order of each group $H_{z}$, and $K_{z}$ is archimedeon, and in fact unique up to duals.

$$
\begin{array}{r}
\text { Proof. If } x_{1}, x_{2} \in X_{1} \text { with (say) } \\
x_{2}>x_{1},
\end{array}
$$

we may put

$$
x_{2}-x_{1}=\frac{m}{2^{n}}, m, n \in N, m<2^{n} .
$$

Thus we define

$$
\alpha=\frac{m}{2^{n+z}},
$$

so that 


$$
\xi^{-\alpha 2^{n}} h_{z, x_{1}} \xi^{\alpha 2^{n}}=h_{z, x_{1}}^{p^{m}}
$$

according to (1).

But

$$
\xi^{-\alpha} h_{z, x_{1}} \xi^{\alpha}=h_{z, x_{2}}
$$

again from (1).

(6) and (7) together show that all elements $h_{z, x}$ for fixed $z$ belong to the same archimedean class, showing ${ }_{z}$ to be archimedean.

Clearly analogous results hold for $K_{z}$. Further we deduce from (6) and (7) that

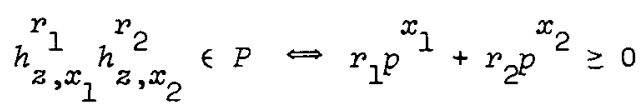

under the condition $h_{z, x_{1}} \in P$, where $p^{x_{1}}$ is a real number taken positive whenever ambiguity might arise.

This follows since $H_{z}$ is archimedean so that it is isomorphic to a subgroup of the real numbers (Fuchs [1], p. 45) whose only automorphisms are given by multiplication by real numbers (Fuchs [1], p. 46); from (6), the number in question for transformation by $\xi^{\alpha}$ is seen to satisfy

$$
(x)^{2^{n}}=p^{m}
$$

so

$$
\begin{aligned}
x & =p^{\frac{m}{2^{n}}} \\
& =p^{x_{2}-x_{1}} .
\end{aligned}
$$

Our result follows by including in the automorphisms the raising to rational powers $r_{1}$ and $r_{2}$. This determines the order of $H_{2}$, while 
its dual occurs if we impose

$$
h_{z, x_{1}} \in-P \text {. }
$$

With the similar results for $K_{z}$, the lemma is proved.

LEMMA 3.11. In any fuzz order of $G(p, q)$, either

$$
\left.\left.\left.|\zeta| \gg\rangle|\xi| \gg \cdots \gg\left|h_{z+n, 0}\right| \gg\right\rangle\left|h_{z+n+1,0}\right| \gg\right\rangle\left|h_{z+n+2,0}\right| \gg\right\rangle \ldots
$$

or

$$
\left.\left.\left.|\zeta| \gg\rangle|\xi| \gg \cdots \gg\rangle\left|h_{z+n, 0}\right| \gg\right\rangle\left|h_{z+n-1}\right| \gg\right\rangle\left|h_{z+n-2,0}\right| \gg\right\rangle \quad \ldots
$$

holds, and either

$$
|\zeta| \gg|\xi| \succ \ldots \gg\left|k_{z+n, 0}\right| \gg|| k_{z+n+1,0}|\gg| k_{z+n+2,0} \mid
$$

or

$$
\left.\left.|\zeta| \gg\rangle|\xi| \gg\rangle \ldots \succ\left|k_{z+n, 0}\right| \gg\right\rangle\left|k_{z+n-1,0}\right| \gg\right\rangle\left|k_{2+n-2,0}\right|
$$

is tme.

Proof. The results follow from the relations

$$
\begin{aligned}
& \xi^{-I_{h, 0}} h_{z, 0}, \\
& \xi^{-I_{z, 0}} k^{p^{2^{z}}}=k_{z, 0}^{q^{2^{z}}}, \\
& \zeta^{-I_{h, 0}} h_{z+1,0},
\end{aligned}
$$

and

$$
\zeta^{-1} k_{z, 0} \zeta=k_{z+1,0}
$$

by applying the preliminary results 2.1 and 2.2 .

We are now able to prove the theorem.

Proof of Theorem 3.1. Since all elements of $G(p, q)$ are uniquely expressible in the form 


$$
\zeta^{a} \xi^{b} n \theta
$$

where $\zeta$ and $\xi$ are as before, and $n \in H, \theta \in K$, Lemma 3.11 ensures that the order of $G(p, q)$ is completely determined by the orders of $Z$, $X, H$ and $K$.

From Lemma 3.10, the "signs" of $h_{z, 0}$ and $k_{z, 0}$ completely determine the order on the groups $H_{z}$ and $K_{z}$ respectively.

From this, the relations

$$
\zeta^{-z} h_{0,0} \zeta^{z}=h_{z, 0}, z \in Z
$$

and

$$
\zeta^{-z} k_{0,0} \zeta^{z}=k_{z, 0}, \quad z \in Z
$$

show that the "sign" of $h_{0,0}$ and $k_{0,0}$ determine the orders of each $H_{z}$ and $K_{z}$. Three cases arise:
(1) $|x| \gg\rangle|y|, x \in H, y \in K$;
(2) $|y| \gg|x|, x \in H, y \in K$;
(3) neither of these hold.

In each of (1) and (2) there are only a finite number of orders possible, $2^{4}$ in all, determined by the choice of "sign" for the elements $\zeta, \xi, h$ and $k_{\text {oo }}$.

$$
\begin{aligned}
& \text { In case (3) there is an integer } m \text { such that either } \\
& \left.\left.\left.\left.\qquad . . \succ\left|h_{0,0}\right| \succ\right\rangle\left|k_{m, 0}\right| \gg\right\rangle\left|h_{1,0}\right| \gg\right\rangle\left|k_{m+1,0}\right| \gg\right\rangle \ldots
\end{aligned}
$$

or

$$
\left.\left.\left.\left.\cdots\rangle\rangle\left|h_{00}\right| \gg\right\rangle\left|k_{m, 0}\right|\right\rangle\right\rangle\left|h_{-1,0}\right| \succ\right\rangle\left|k_{m-1,0}\right| \gg \ldots
$$

depending on the possibilities of Lemma 2 .

There are countably many such choices of ordering and since the first two cases give only 32 orders, the theorem is proved. 


\section{Reference}

[1] L. Fuchs, Partially ordered algebraic systems (Pergamon Press, Oxford, London, New York, Paris, 1963).

University of Queensland,

St Lucia,

Queensland. 